\title{
Seroprevalencia de Neospora caninum en Bovinos de una Feria Ganadera de la Región de la Araucanía, Chile
}

\author{
Seroprevalence of Neospora caninum in Cattle in a Livestock Fair in the \\ Araucania Region, Chile \\ Christian Tuemmers ${ }^{1}$, Gastón Valenzuela ${ }^{1}$, Christian Nuñez ${ }^{1}$, Ricardo De la Cruz ${ }^{1}$, \\ Jorge Meyer ${ }^{1}$, Marcela Andaur ${ }^{2}$, Pilar Leyan ${ }^{2}$, Carolina Mora ${ }^{1}$
}

\section{Resumen}

El objetivo del estudio fue determinar la seroprevalencia de neosporosis en ganado bovino llevado a una feria ganadera en la Región de La Araucanía, Chile. Se muestrearon 437 vacas y vaquillas procedentes de predios ganaderos de comunas cercanas. Las muestras de suero fueron analizadas mediante la técnica ELISA indirecta para detectar anticuerpos contra Neospora caninum. El 21.1\% (92/437) de las muestras resultaron positivas a $N$. caninum, mientras que $13.9 \%(61 / 437)$ resultó sospechoso y $65.0 \%(284 / 437)$ resultó negativo Los resultados obtenidos indican que la infección se encuentra diseminada en los predios y comunas de la región.

Palabras clave: Neospora caninum; bovino; aborto; seroprevalencia

\section{AbSTRaCT}

The aim of the study was to determine the seroprevalence of neosporosis in cattle in a livestock fair in the Region of La Araucanía, Chile. Blood samples were collected in 437 cows and heifers belonging to nearby farms. Serum samples were analyzed by the indirect ELISA technique to detect antibodies against Neospora caninum. The results showed that $21.1 \%$ of the animals were positive to neosporosis, while $13.9 \%(61 / 437)$ was uncertain and $65.0 \%(284 / 437)$ was negative. The results indicate that the infection is spread in the farms of the region.

Key words: Neospora caninum; bovine; abortion; seroprevalence

\footnotetext{
${ }^{1}$ Escuela de Medicina Veterinaria, Facultad de Recursos Naturales, Universidad Católica de Temuco, Chile

${ }^{2}$ Escuela Ciencias de la Salud, Universidad Católica de Temuco, Chile

${ }^{3}$ E-mail: ctuemmers@uct.cl
}

Recibido: 24 de noviembre de 2016

Aceptado para publicación: 10 de mayo de 2017 


\section{INTRODUCCIÓN}

La neosporosis bovina es una enfermedad parasitaria emergente, que ha adquirido importancia global como una de las principales causas de aborto en el ganado bovino (Dubey et al., 2007). La enfermedad ocasiona subfertilidad, pérdidas tempranas de gestación, momificaciones, abortos y nacimiento de terneros con ataxia y parálisis. La enfermedad es producida por el parásito Neospora caninum, protozoo parásito intracelular, de la familia Sarcocystidae, phylum Apicomplexa, y está estrechamente relacionado con Toxoplasma (Barr et al., 1997; Cordero y Rojo, 1999; Dubey, 2003). Los hospederos definitivos de $N$. caninum conocidos hasta ahora son el perro (Canis familiaris) (McAllister et al., 1998) y el coyote (Canis latrans) (Dubey et al., 2004; Gondim et al., 2004).

La neosporosis puede ocasionar la muerte del embrión o del feto y su reabsorción. El aborto tiene lugar entre el tercer y el noveno mes de la gestación, siendo más frecuente en torno a los cuatro y siete meses (Anderson et al., 1995; Campero et al., 1998). La probabilidad de muerte fetal después de una infección por $N$. caninum es mayor en los fetos jóvenes (Williams et al., 2000; Innes et al., 2002).

El hospedero definitivo elimina ooquistes contaminando praderas, alimentos y agua. Los hospederos intermediarios se infectan al ingerir el pasto y agua contaminado (Patitucci et al., 2001); sin embargo, una de las principales vías de transmisión y persistencia de la infección en el bovino es la vía vertical (Maley et al., 2003; Piergili et al., 2003), donde la infección transplacentaria endógena es la que ocurre con mayor frecuencia (Dijkstra et al., 2008). Daft et al. (1996) reportan que más del $80 \%$ de las vacas seropositivas transmiten la infección a su descendencia, dándole poca importancia a la infección posnatal. En los bovinos adultos, el aborto es el único signo clínico observado, aunque la mayor parte de las vacas son capaces de controlar la infección y permanecer clínicamente asintomáticas, aunque persistentemente infectadas. Los abortos se pueden presentar en forma aislada o en brotes epidémicos (Cole et al., 1995; Dubey, 1999).

El diagnóstico de aborto por neosporosis es complejo, ya que la serología positiva sólo es indicativa de exposición a $N$. caninum. Se han desarrollado una serie de técnicas diagnósticas, siendo las más utilizadas la técnica de Ensayo por Inmunoabsorción Ligado a Enzimas (ELISA) e Inmunofluorescencia Indirecta (IFI). El ELISA ha demostrado ser una técnica con alta sensibilidad y especificidad (von Blumröder et al., 2004), y es además la técnica de referencia que utiliza el Servicio Agrícola y Ganadero del país.

En Chile, las pérdidas ocasionadas por los abortos bovinos no han sido del todo cuantificadas, pero son económicamente importantes. Más del $50 \%$ de los episodios abortigénicos son de etiología desconocida y, de los diagnosticados, la mayoría corresponden a causas infecciosas (Patitucci et al., 1999). Estos investigadores evaluaron establecimientos lecheros de la IX Región demostrando la presencia de anticuerpos contra $N$. caninum en vacas que presentaron abortos y mediante el uso de la inmunohistoquímica confirmaron la enfermedad en el país. El objetivo del presente estudio fue determinar el estado actual de la seroprevalencia de neosporosis en hembras bovinas ingresadas en una feria ganadera local, donde llegan en promedio 300 bovinos por día de feria procedentes de diversas localidades de la región de la Araucanía.

\section{Materiales y Métodos}

La investigación correspondió a un estudio descriptivo de corte transversal con muestreo aleatorio y se llevó a cabo en la Feria Ganadera Traiguén Ltda., ubicada en la comuna de Traiguén, provincia de Malleco, 
región de la Araucanía (Chile), a la cual llega ganado bovino de sectores y comunas cercanas como Los Sauces, Angol, Renaico, Purén, Lumaco, Cañete, Lebu, Tirúa, Arauco, Carahue, Puerto Saavedra y Galvarino.

La colección de muestras de sangre de los animales (437 vacas y vaquillas) se realizó en seis visitas con un rango de 70 a 75 animales por visita entre los meses de enero y febrero del 2015, registrándose, además, la raza, edad y origen de los animales. La edad de los animales fue estimada por cronometría dentaria ( 0 dientes: $<1$ año; 2 dientes: 2.5 años; 4 dientes: 3.5 años; 6 dientes: 4.5 años; 8 dientes: $>5$ años).

Las muestras de sangre se tomaron por punción de la vena coccígea caudal, utilizando tubos al vacío (sistema vacutainer) de 4 $\mathrm{ml}$ sin anticoagulantes. Las muestras fueron centrifugadas a $1008 \mathrm{~g}$ por 5 minutos y el suero resultante fue almacenado a $-18{ }^{\circ} \mathrm{C}$. Los sueros se analizaron mediante la prueba comercial CIVTEST Bovis Neospor ${ }^{\mathrm{TM}}$ (Hipra), basada en un ELISA indirecto, con antígeno específico de $N$. caninum. La lectura se realizó con el lector de microplacas Rayto RT $2100^{\mathrm{TM}}$ midiendo la absorbancia a $405 \mathrm{~nm}$. Se consideró como test válido si la densidad óptica (DO) media del control positivo era $>0.9$ y la relación DO media del control positivo y la DO media del control negativo era $>5.0$.

El Índice Relativo x 100 (IRPC) de cada muestra se obtuvo de la diferencia entre el DO de la muestra de la media DO del control negativo dividido entre la diferencia de la media DO del control positivo y la media DO del control negativo. Las muestras de suero con cocientes IRPC $<6.0,>6.0 \mathrm{y}<10, \mathrm{y}>10.0$ se consideraron como negativas, sospechosas y positivas a anticuerpos contra Neospora, respectivamente.

El análisis de los datos se realizó mediante estadística descriptiva a través del programa SPSS v. 19 para Windows (SPSS Inc., Chicago IL, EEUU).

\section{Resultados y Discusión}

El 21.1\% (92/437) de las muestras resultaron positivas a $N$. caninum, mientras que $13.9 \%$ (61/437) resultó sospechoso y $65.0 \%$ (284/437) resultó negativo.

El porcentaje de seropositividad es similar al 23\% reportado por MondragónZavala et al. (2011) en México y superior al $17.8 \%$ en predios brasileros donde existió convivencia con perros (Corbellini et al., 2006). No obstante, se dispone de reportes con seroprevalencias entre 42 y $59 \%$ en ganado lechero de México (García-Vázquez et al., 2002, 2005) y hasta de $72 \%$ en Coahuila y Querétaro, México en sistemas lecheros intensivos (Morales et al., 2001).

El mayor porcentaje de animales que ingresó a la feria fue de doble propósito (Clavel Alemán, Holando Europeo y mestizas). Por otro lado, considerando el número de animales muestreados, las mayores frecuencias de seropositividad se encontraron en animales Hereford (36.4\%), Angus Rojo

Cuadro 1. Hembras bovinas, según raza, seropositivas a Neospora caninum (Región de la Araucanía, Chile, 2015)

\begin{tabular}{lccc}
\hline \multirow{2}{*}{ Raza } & Total de & \multicolumn{2}{c}{ Positivos } \\
\cline { 3 - 4 } & bovinos & $\mathrm{n}$ & $\%$ \\
\hline Angus Negro & 1 & 0 & 0 \\
Angus Rojo & 25 & 9 & 36.0 \\
Charolais & 47 & 8 & 17.0 \\
Clavel Alemán & 172 & 35 & 20.3 \\
Hereford & 11 & 4 & 36.4 \\
Holando & 61 & 14 & 23.0 \\
Europeo & 1 & 0 & 0 \\
Limousin & 111 & 19 & 17.1 \\
Mestiza & 8 & 3 & 37.5 \\
Normando & 437 & 92 & 21.1 \\
\hline Total & & &
\end{tabular}




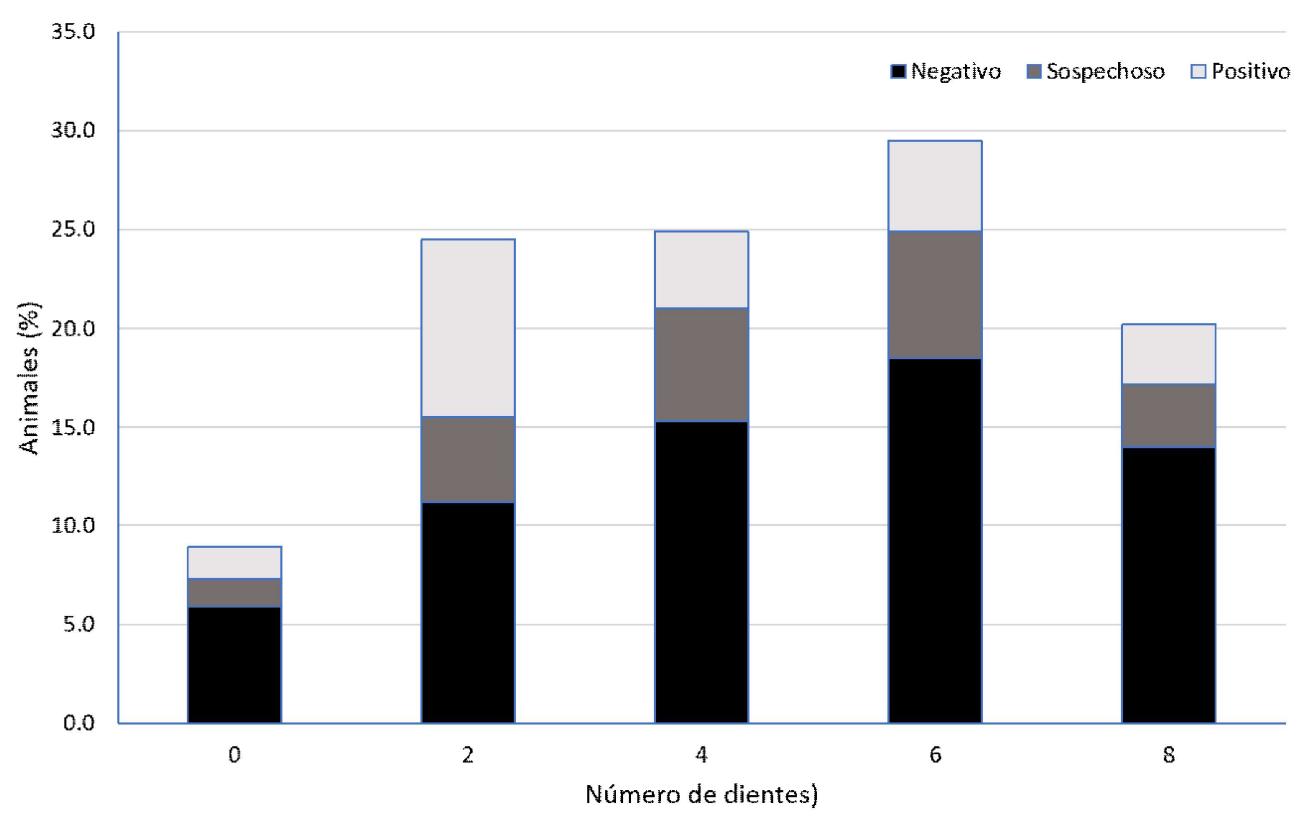

Figura 1. Distribución porcentual de hembras bovinas según edad y seropositividad a Neospora caninum (Región de la Araucanía, Chile, 2015)

(36.0) y Normando (37.5\%), mientras que en animales mestizos se tuvo $17.1 \%$ de seropositividad (Cuadro 1). En estudios realizado en Senegal (Innes et al., 2002) y México (Romero-Salas et al., 2010) se reporta un mayor riesgo y frecuencia de animales mestizos seropositivos en relación a razas exóticas, mientras que en Sudán no se detectaron diferencias en seropositividad entre razas locales y cruces (Ibrahim et al., 2012). Existen evidencias de que la prevalencia de la neosporosis varía entre ganado lechero y ganado de carne, observándose menores niveles de prevalencia en el ganado de carne (Moore et al., 2002; Bartels et al., 2006; Armengol et al., 2006), así como menores riesgos de aborto (De Meerschman et al., 2000); aspecto que no pudo ser corroborado en este estudio donde el ganado Hereford y el Angus Rojo presentaron las mayores prevalencias.
Cuadro 2. Hembras bovinas, según localidad de origen, seropositivas a Neospora caninum (Región de la Araucanía, Chile, 2015)

\begin{tabular}{lccc}
\hline \multirow{2}{*}{ Localidad } & Total de & \multicolumn{2}{c}{ Positivos } \\
\cline { 3 - 4 } & bovinos & $\mathrm{n}$ & $\%$ \\
\hline Angol & 39 & 5 & 12.8 \\
Arauco & 8 & 2 & 25.0 \\
Cañete & 29 & 6 & 20.7 \\
Carahue & 7 & 2 & 28.6 \\
Galvarino & 13 & 0 & 0 \\
Lebu & 10 & 5 & 50.0 \\
Los Álamos & 25 & 3 & 12.0 \\
Los Sauces & 136 & 36 & 26.5 \\
Lumaco & 4 & 0 & 0 \\
Pto Saavedra & 9 & 4 & 44.4 \\
Purén & 47 & 6 & 12.8 \\
Renaico & 9 & 2 & 22.2 \\
Tirúa & 10 & 0 & 0 \\
Traiguén & 91 & 21 & 23.1 \\
\hline Total & 437 & 92 & 21.1 \\
\hline
\end{tabular}


De las 437 muestras analizadas, 327 correspondieron a vacas y 110 a vaquillas. El análisis en las vacas (4, 6, 8 dientes) se encontró 68 positivas, 50 sospechosas y 209 negativas, en tanto que en las vaquillas ( 0 , 2 dientes) hubieron 24 positivas, 11 sospechosas y 75 negativas (Figura 1). Los resultados indican seroprevalencias similares para vaquillas $(21.8 \%, 24 / 110)$ y vacas $(20.8 \%$, $68 / 327$ ), en concordancia con los resultados presentados por Escalona et al. (2010). Por otro lado, Davison et al. (1999) y GarcíaVázquez et al. (2005) encontraron una menor seroprevalencia en animales jóvenes en comparación con aquella en animales de mayor edad.

El origen de los animales muestreados y su estado sanitario para neosporosis se muestra en el Cuadro 2. El mayor número de animales provenía de las comunas más cercanas a la feria, tales como Los Sauces y Traiguén. No obstante, las mayores seroprevalencias se encontraron en animales procedentes de las comunas de Lebu $(50 \%)$ y Puerto Saavedra (44.4\%), aunque el número de animales muestreados fue relativamente bajo. El mayor número de animales procedió de Los Sauces $(\mathrm{n}=36)$, donde la seroprevalencia fue de $26.5 \%$ (Cuadro 2).

No se pudo conocer el tamaño poblacional de los predios de donde procedía el ganado en estudio, pero, aparentemente, hay mayor riesgo de exposición en los rebaños más grandes que en los más pequeños (Otranto et al., 2003; Schares et al., 2004).

\section{ConClusiones}

Los valores de seropositividad evidencian la presencia de anticuerpos antiNeospora caninum, indicando que la enfermedad está presente en un área estratégica para la producción ganadera en la IX región de la Araucanía, constituyendo un riesgo potencial para su eficiencia reproductiva.

\section{Literatura Citada}

1. Anderson ML, Palmer CW, Thurmond MC. 1995. Evaluation of abortions in cattle attributable to neosporosis in selected dairy herds in California. J Am Vet Med Assoc 207: 1206-1210.

2. Armengol R, Pabón M, Adelantado C, López-Gatius F, Almeria S. 2006. First report of Neospora caninum abortion in a beef cow-calf herd from Andorra, Europe. J Parasitol 92: 1361-1362. doi: 10.1645/GE-949R.1

3. Barr B, Bjerkas I, Buxton D, Conrad P, Dubey J, Ellis J, Jenkins M, et al. 1997. Neosporosis: report of the international Neospora workshop. Comp Cont Educ Pract 19: 120-126.

4. Bartels CJ, Arnaiz-Seco JI, Ruiz-Santa-Quitera A, Björkman C, Frössling J, von Blumröder D, Conraths FJ, et al. 2006. Supranational comparison of Neospora caninum seroprevalences in cattle in Germany, The Netherlands, Spain and Sweden. Vet Parasitol 137: 1727. doi: 10.1016/j.vetpar.2005.12.016

5. Campero CM, Anderson ML, Conosciuto $G$, Odriozola $H$, Bretschneider G, Poso MA. 1998. Neospora caninum-associated abortion in a dairy herd in Argentina. Vet Rec 143: 228-229.

6. Corbellini LG, Smith RD, Pescador CA, Schmitz M, Correa U, Steffen DJ, Driemeier D. 2006. Herd-level risk factors for Neospora caninum seroprevalence in dairy farms in southern Brazil. Prev Vet Med 74: 130-141. doi: 10.1016/j.prevetmed.2005.11.004

7. Cordero del Campillo M, RojoVásquez F. 1999. Parasitología veterinaria. México DF: McGraw- Hill Interamericana. $968 \mathrm{p}$.

8. Cole R, Lindsay DS, Blagburn BL, Dubey JP. 1995. Vertical transmission of Neospora caninum in mice. J Parasitol 81: 730-732. 
9. Daft BM, Barr B, Collins N, Sverlow K. 1996. Neospora encephalomyelitis and polyradiculoneuritis in an aged mare with Cushing's disease. Equine Vet J 28: 240-243. doi: 10.1111/j.20423306.1997.tb01678.x

10. Davison HC, Otter A, Trees A. 1999. Estimation of vertical and horizontal transmission parameters of Neospora caninum infections in dairy cattle. Int $\mathrm{J}$ Parasitol 29: 1683-1689. doi: 10.1016/ S0020-7519(99)00129-0\}

11. De Meerschman F, Focant C, Boreux R, Leclipteux T, Losson B. 2000. Cattle neosporosis in Belgium: a case-control study in dairy and beef cattle. Int $\mathbf{J}$ Parasitol 30: 887-890.

12. Dijkstra T, Lam TJ, Bartels CJ, Eysker M, Wouda W. 2008. Natural postnatal Neospora caninum infection in cattle can persist and lead to endogenous transplacental infection. Vet Parasitol 152: 220-225. doi: 10.1016/ j.vetpar.2007.12.034

13. Dubey JP. 1999. Recent advances in Neospora and neosporosis. Vet Parasitol 84: 349-367. doi: 10.1016/S03044017(99)00044-8

14. Dubey J. 2003. Review of Neospora caninum and neoporosis in animals. Korean J Parasitol 41:1-16. doi: 10.3347/ kjp.2003.41.1.1

15. Dubey J, Sreekumar C, Knickman E, Miska K, Vianna M, Kwok O, Hill D, et al. 2004. Biologic, morphologic, and molecular characterisation of Neospora caninum isolates from littermate dogs. Int J Parasitol 34: 1157-1167. doi: 10.1016/j.ijpara.2004.07.005

16. Dubey J, Schares G, Ortega-Mora L. 2007. Epidemiology and control of neosporosis and Neospora caninum. Clin Microbiol Rev 20: 323-367. doi: 10.1128/CMR.00031-06

17. Escalona J, García F, Mosquera O, Vargas F, Corro A. 2010. Factores de riesgo asociados a la prevalencia de neosporosis bovina en el municipio Bolívar del estado Yaracuy, Venezuela. Zootecnia Trop 28: 201-212.
18. García-Vázquez Z, Cruz-Vázquez C, Medina Espinoza L, García-Tapia D, Chavarria Martínez B. 2002. Serological survey of Neospora caninum infection in dairy cattle herds in Aguascalientes, Mexico. Vet Parasitol 106: 115-120. doi: 10.1016/S03044017(02)00040-7

19. García-Vázquez Z, Rosario-Cruz $R$, Ramos-Aragón A, Cruz-Vázquez C, Mapes-Sánchez G. 2005. Neospora caninum seropositivity and association with abortions in dairy cows in Mexico. Vet Parasitol 134: 61-65. doi: 10.1016/ j.vetpar.2005.07.007

20. Gondim LF, McAllister M, Pitt W, Zemlicka D. 2004. Coyotes (Canis latrans) are definitive hosts of Neospora caninum. Int J Parasitol 34: 159-161. doi: 10.1016/j.ijpara.2004.01.001

21. Ibrahim AM, Elfahal AM, EL Hussein AR. 2012. First report of Neospora caninum infection in cattle in Sudan. Trop Anim Health Prod 44: 769-772. doi: 10.1007/s11250-011-9963-5

22. Innes EA, Andrianarivo $A G$, Björkman C, Williams DJ, Conrad PA. 2002. Immune responses to Neospora caninum and prospects for vaccination. Trends Parasitol 18: 497-504. doi: 10.1016/S1471-4922(02)02372-3

23. McAllister MM, Dubey JP, Lindsay DS, Jolley WR, Wills RA, McGuire AM.1998. Dogs are definitive hosts of Neospora caninum. Int J Parasitol 28: 1473-1478. doi: 10.1016/S00207519(98)00138-6

24. Maley SW, Buxton D, Rae AG, Wright SE, Schock A, Bartley PM, EstebanRedondo I, et al. 2003. The pathogenesis of neosporosis in pregnant cattle: inoculation at mid-gestation. J Comp Path 129: 186-195. doi: 10.1016/ S0021-9975(03)00032-X

25. Mondragón-Zavala K, Cruz-Vázquez C, Medina-Esparza L, Ramos-Parra M, García-Vázquez Z. 2011. Infección por Neospora caninum en ganado de carne mantenido en condiciones de pas- 
toreo en el centro-norte de México. Rev MVZ Córdoba 16: 2484-2490.

26. Morales E, Trigo F, Ibarra F, Puente E, Santacruz M. 2001. Neosporosis in Mexican dairy herds: lesions and immunohistochemical detection of Neospora caninum in fetuses. J Comp Pathol 125: 58-63. doi: 10.1053/ jcpa.2001.0477

27. Moore DP, Campero CM, Odeón AC, Poso MA, Cano D, Leunda MR, et al. 2002. Seroepidemiology of beef and dairy herds and fetal study of Neospora caninum in Argentina. Vet Parasitol 107: 303-316. doi: 10.1016/S03044017(02)00129-2

28. Otranto D, Llazari A, Testini G, Traversa $D$, Frangipane Di Regalbono A, Badan CM, Capelli $G$ 2003. Seroprevalence and associated risk factors of neosporosis in beef and dairy cattle in Italy. Vet Parasitol 118: 718. doi: 10.1016/j.vetpar.2003.10.008

29. Piergili $\mathrm{D}_{2}$ PasqualI P, Diaferia $M$, Mangili V, Rosignoli L. 2003. Neospora caninum infection and congenital transmission: serological and parasitological study of cows up to the fourth gestation. J Vet Med B 50: 399404. doi: $10.1046 / j .1439-0450.2003 .-$ 00686.x

30. Patitucci A, Pérez M, Luders C, Ratto M, Dumont A. 1999. Evidencia serológica de infección por Neospora caninum en rebaños lecheros del sur de Chile. Arch Med Vet 31: 215-218. doi: 10.4067/S0301-732X1999000200009

31. Patitucci AN, Phil M, Pérez MJ, Rosas MA, Israel KF. 2001. Neosporosis canina: presencia de anticuerpos séricos en poblaciones caninas rurales y urbanas de Chile. Arch Med Vet 33: 227-232. doi: 10.4067/S0301-732X2001000200011

32. Reichel M, Ellis J, Dubey P. 2007. Neosporosis and hammondiosis in dogs. J Small Anim Pract 48: 308-312. doi: 10.1111/j.1748-5827.2006.00236.x

33. Romero-Salas D, García-Vázquez Z, Montiel-Palacios F, Montiel-Peña T, Aguilar-Domínguez, M, Medina-Esparza, Cruz-Vázquez. L. 2010. Seroprevalence of Neospora caninum antibodies in cattle in Veracruz, Mexico. J Anim Vet Adv 9: 1445-1451. doi: 10.3923/javaa.2010.1445.1451

34. Schares G, Bärwald A, Staubach C, Ziller M, Klöss D, Schröder R, Labohm R, et al. 2004. Potential risk factors for bovine Neospora caninum infection in Germany are not under the control of the farmers. J Parasitol 129: 301-309. doi: 10.1017/S0031182004005700

35. von Blumröder D, Schares G, Norton R, Williams DJ, Esteban-Redondo I, Wright S, Björkman C, et al. 2004. Comparison and standardization of serological methods for the diagnosis of Neospora caninum infection in bovines. Vet Parasitol 120: 11-22. doi: 10.1016/ j.vetpar.2003.12.010

36. Williams DJ, de Guy CS, Mcgarry JW, de Guy F, Tasker L, Smith RF, MacEachern K, et al. 2000. Neospora caninum-associated abortion in cattle: the time of experimentally-induced parasitaemia during gestation determines foetal survival. Parasitol 121: 347-358. 